\title{
Automated, high-throughput immunofluorescence staining: a new approach
}

\author{
J. L. J. van de Bilt • J. H. J. Derks • J. D. Janse
}

Received: 11 January 2007 / Accepted: 19 July 2007 / Published online: 11 September 2007

(C) KNPV 2007

\begin{abstract}
A high-throughput antibody staining and washing system for large-scale routine indirect immunofluorescence testing was developed, using a robotic sample processor. The Tecan robotic sample processor (Miniprep 75/2, Tecan Benelux BV, Giessen, The Netherlands) was introduced, adapted and evaluated for its suitability for large-scale immunofluorescence screening of potato samples for the presence of the plant pathogenic quarantine bacterium, Ralstonia solanacearum. Throughput and quality of antibody-antigen reactions were compared to manual sample preparation for the immunofluorescence test according to a standard EU testing protocol, using 216 standard potato extracts, including 204 known negative extracts and 12 known positive extracts, and 24 positive control strain samples. Throughput of the Tecan robotic sample processor easily outcompeted manual staining of immunofluorescence slides. The robot was twice as fast as manual processing and is easily cost effective. With the robot 288 samples per day can be processed as compared to 144 samples per day with manual processing. No difference between quality of antibody-antigen reactions and readability of prepara-
\end{abstract}

J. L. J. van de Bilt · J. H. J. Derks · J. D. Janse $(\bowtie)$ Department of Bacteriology, Plant Protection Service, Geertjesweg 15, P.O. Box 9102, 6700 HC Wageningen, The Netherlands e-mail: j.d.janse@minlnv.nl e-mail: jjanse@nak.nl tions under a microscope could be detected. The new Tecan robotic sample processor was found to be useful for high-throughput fluorescent antibody staining and washing in large-scale, routine indirect immunofluorescence testing.

Keywords Automation Clavibacter michiganensis subsp. sepedonicus $\cdot$ Ralstonia solanacearum .

Robotic sample processor $\cdot$ Routine testing

Following an outbreak of brown rot, caused by Ralstonia solanacearum (Rsol) race 3 biovar 2 in potato (Solanum tuberosum) in 1995 in the Netherlands, the Dutch Plant Protection authorities started an intensive eradication programme. This programme also included testing for the presence of latent infections of brown rot of all seed potato lots intended for marketing. From 1989 onwards several other European countries have experienced outbreaks of brown rot in potato. In the same period Clavibacter michiganensis subsp. sepedonicus (Cms), causing bacterial ring rot, was reported in several European countries, often leading to large scale screening of potato seeds in these countries (Schans and Steeghs 1998; Janse 1996).

Screening for latent infections of $\mathrm{Rsol}$ and $\mathrm{Cms}$ in potato is performed in the EU by indirect immunofluorescence (IIF), following official European standard testing protocols (see: http://eur-lex.europa.eu/ LexUriServ/LexUriServ.do?uri=CELEX:32006L0056: EN:NOT and http://eur-lex.europa.eu/LexUriServ/Lex- 
UriServ.do?uri=CELEX:32006L0063:EN:NOT). The EU protocol involves application of four antibody dilutions to sample preparations fixed in separate windows of immunofluorescence slides to determine the end-point titre of the antibody for each sample in which fluorescing bacterial cells are detected and to enable better evaluation of cross-reactive cells. The Dutch Plant Protection Service (PPS) and the Dutch Seed Inspection (NAK) have performed large-scale IIF screening for latent infections of potato brown rot $(\mathrm{Rsol})$ and ring rot $(\mathrm{Cms})$ with manual sample preparation since 1995 . Annually c. 35-70,000 samples are processed of which the majority is tested between September and December each year, resulting in c. 500-1,000 samples of 200 tubers per day. For this large-scale screening, automation of some of the IF slide processing steps could save time and labour costs.

Because of high-throughput perspectives and reasonable pricing we contracted the Tecan B.V. compa- ny (Giessen, NL) and adapted their robotic sample processor (Miniprep 75/2) and Gemini software to the IF staining procedure. In this contribution we report on results of evaluation of the robot as compared to manual staining of IIF microscopic slide preparations.

The IIF-screening of potato lots, involving sample preparation and the IIF technique using a polyclonalspecific serum from rabbit (Loewe Biochemica $\mathrm{GmbH}$, Sauerlach, DE), and swine/anti-rabbit conjugated with fluorescein isothiocyanate (Nordic Immunological Laboratories, Tilburg, NL), was performed following entirely the European standard protocol with slide preparation according to procedure 5.1.i http://eur-lex.europa.eu/LexUriServ/LexUriServ.do? uri=CELEX:32006L0056:EN:NOT), also see Fig. 1. The Tekan Miniprep 75/2 is a double-armed smallfootprint robotic sample processor (see Fig. 2), of which each arm is equipped with an eight-channel manifold. The system is controlled by Gemini Mini-
Fig. 1 Summary of IFscreening as routinely performed. The grey areas depict the steps that were automated using the Tecan Miniprep 75/2 sample processor

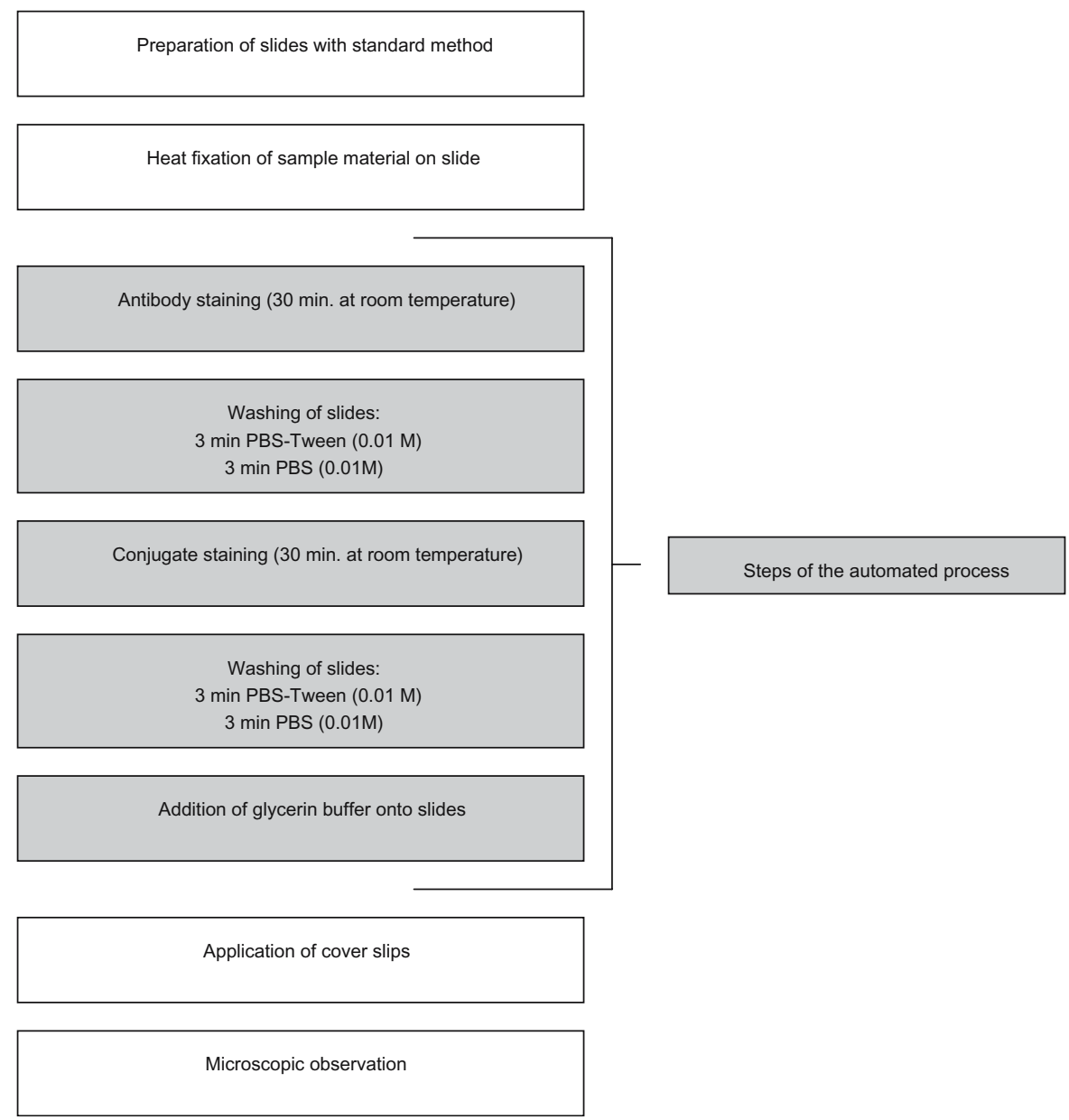




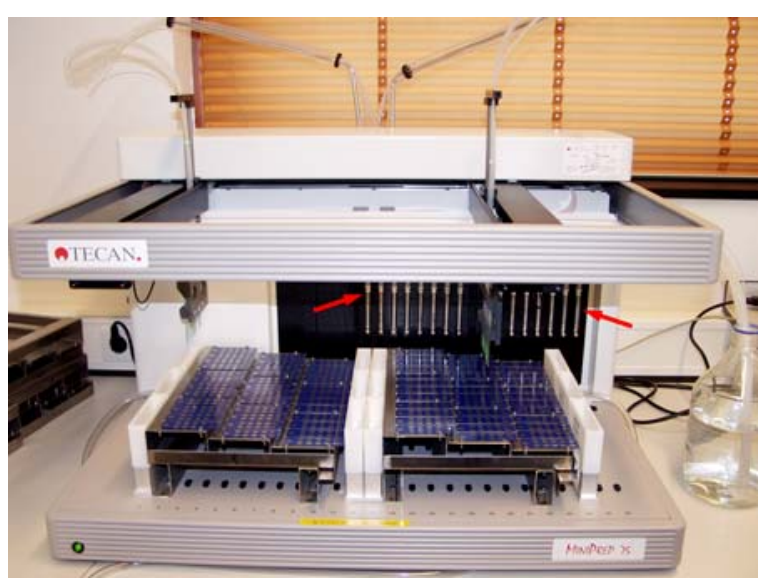

Fig. 2 Tecan Miniprep 75/2 robotic sample processor, showing two work areas with 36 IF slides each and two robotic arms with 8 pipette tips each (red arrows)

prep 3.31 application software. To keep implications for hardware and programming as basic as possible, focus in this study was on one pathogen, namely Rsol. With the Tecan company the Tecan Miniprep 75/2 robotic sample processor was adjusted to the IF procedure. The working area of the automated system was divided into two parts (Fig. 3). For each part a special rack for carrying slides was designed according to our specifications. The two racks can hold 72 samples, each rack 9 groups of 4 slides. Each robotic arm processes one side of the working area. The five antiserum dilutions and conjugate are applied in reservoirs in the centre of the working area. Both robotic arms use these reservoirs. The PBS-Tween washing liquid and the glycerine buffer are supplied from reservoirs on the outside perimeter of the working area and used by the corresponding arm only. The PBS washing liquid is used as system liquid and is therefore supplied in bulk from outside the working area (Fig. 3).

Robotic treatment of the slides involved the administration of antiserum to the individual windows in each slide up to and including the administration of glycerine buffer (Fig. 1). Drops of liquid (serum or buffer) are applied to, or removed from, individual windows per sample preparation and because the eight-channel manifold does this, eight samples (i.e. four slides at two samples per slide) can be processed at a time. Drops of antiserum dilutions are applied separately per dilution step to all slides. Both arms work independently and at the same time. Application and removal of all liquids are performed per group of four slide samples, except application of antiserum (applied per dilution to all slides for one arm). The standard robot arm was adapted in its channel distance in order to match with the windows of the microscope

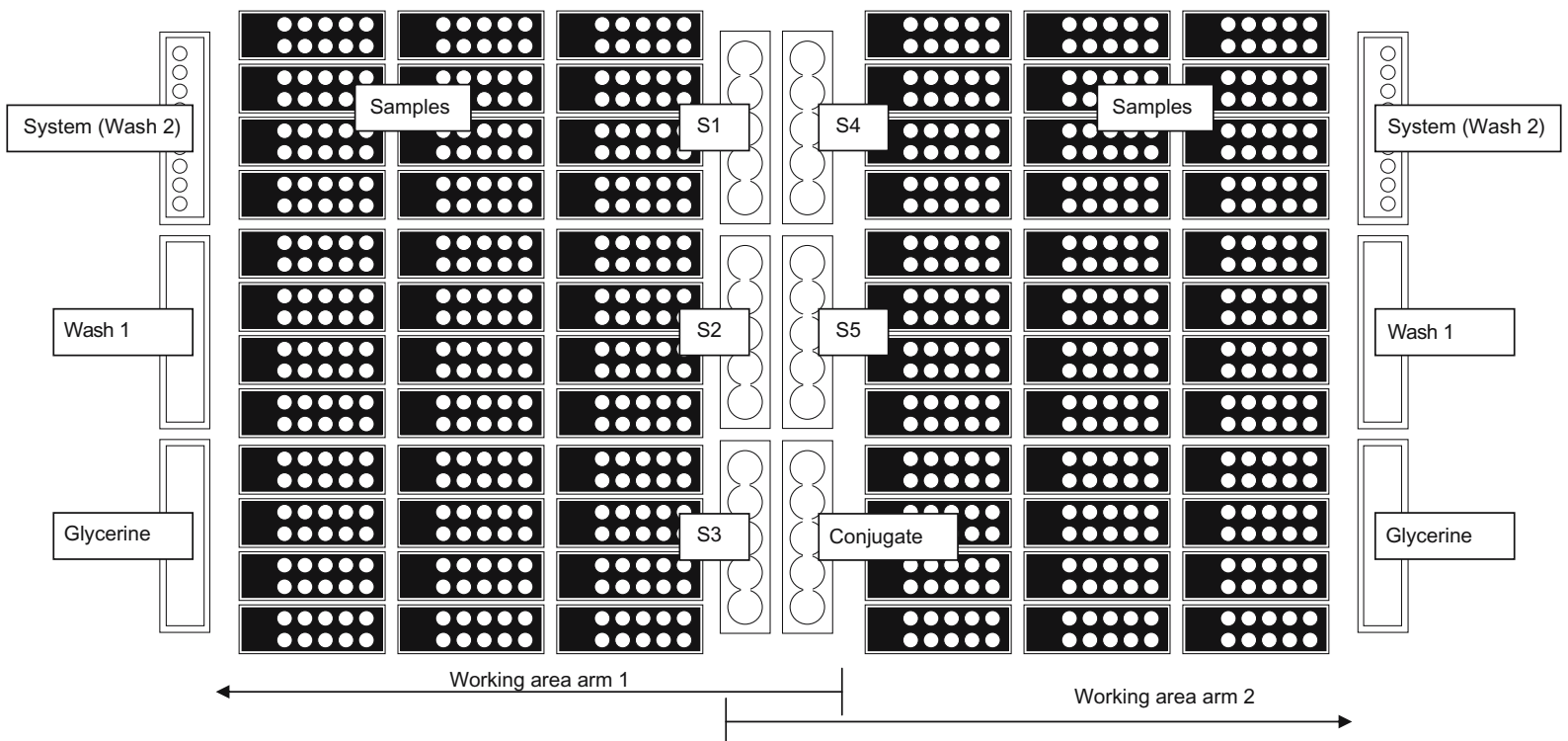

Fig. 3 Overview of the working area of the Tecan Miniprep $75 / 2$ robotic sample processor: $S 1$ serum dilution $1(1: 1,600)$; $S 2$ serum dilution $2(1: 3,200) ; S 3$ serum dilution 3 (1:6,400); $S 4$ serum dilution 4 (1:12,800); $S 5$ serum dilution $5(1: 25,600)$; Conjugate conjugate SwAR/FITC; Wash 1 washing buffer
PBS-Tween (0.01 M PBS with 0.1\% Tween 20); System (Wash 2) system liquid and washing buffer $0.01 \mathrm{M}$ PBS; Glycerine phosphate glycerine buffer; Samples nine groups of four IF slides (one row of five wells per sample) 
slide. To assure that the windows are completely covered with antiserum, the liquid drops are spread over the window by a slight side-shifting of the robotic arm while the pipette tips are still in touch with the drops of liquid. This side-shifting, however, proved not to be necessary in the washing steps due to automatic spreading of the $50 \mu$ l fluid per window.

The most important difference in treatment of the slides as compared to the manual treatment is the washing method, since the automated system can only add and remove drops of liquid and cannot dip slides into a washing bath. Simply applying and removing washing liquids up to $50 \mu \mathrm{l}$ per window proved not to be sufficient. In this case a uniform green-fluorescent background film was observed as well as a huge amount of non-specific green specks in and especially around the perimeter of the windows. The presence of these specks lengthened the time needed for IF microscopic examination. After optimization we found that three washing steps, of 3 min each (two times $50 \mu \mathrm{l}$ PBS-Tween followed by one time PBS) and drying the slides with filter paper reduced this undesirable effect with a minimum of work. The throughput capacity for one complete treatment with a maximum number of slides, from now on referred to as a 'run', is 72 microscope slides (144 samples), i.e. 36 microscope slides per arm. It was possible to treat 288 slides (576 samples) in 1 day. Another necessary hardware adaptation was the construction of a dark plexiglass hood over the machine to enable incubation of slides in the dark, and to increase humidity during the incubation to avoid drying of the droplets on the slide.

The Tecan miniprep 75/2 robot was subsequently tested by us with regard to quality, throughput and robustness where special attention was given to cell numbers, fluorescence intensity and cell shape (typical or a-typical). For robustness and throughput we set as a criterion that we should be able to work 3 days at maximum capacity (288 slides per day) without technical problems.

The following samples were used: negative samples (NS), positive samples (PS), positive control samples (PC), and empty sample slides (ES). NS were routine samples from the potato testing programme. PS slides were negative potato samples spiked with a suspension of a 3-day-old culture (nutrient agar) of Rsol biovar 2, race 3 strain $\mathrm{PD} 2762$ from potato $(\mathrm{PD}=$ Culture Collection of the Dutch Plant Protection Service) in phosphate buffer $0.01 \mathrm{M}, \mathrm{pH}$ 7.2). PS had three different contamination levels (low, medium and high), c. 10 , c. $10^{2}$ and c. $10^{3}$ typical fluorescent cells per field of view, respectively. PC slides were prepared with a similar bacterial culture as described for PS slides, suspended in tap water with PBS Tween 20 to a final concentration of Tween of c. $0.004 \%$ (c. 50 cells per field of view). Each run contained $17 \mathrm{NS}$, $1 \mathrm{PS}$ and 2 PC slides. PC slides were always put in the same position, one at each side of the working area. NS and PS slides were divided in two groups of nine slides and randomly positioned on the left and right rack, based on SigmaPlot 4.0 statistical software determination. The remaining 52 positions per rack were filled with ES slides. The experiment lasted three testing days, and utilized 204 NS, 12 PS, 24 PC and 624 ES slides. A control group of the same samples (204 NS, 12 PS and 12 PC slides) were stained manually. PC slides were scored for a mean fluorescence intensity (FI) of cells per window only. PS and NS slides were scored per window for FI, number of cells, and for cell morphology. The same person examined the samples from the robotic and the manual treatments in a comparative manner, i.e. observation of the manually treated slide was followed directly by observation of a slide with the same samples from the automated robotic procedure. Also differences in nonspecific background staining and general appearance of the sample extracts were noted.

The quality of the slides, treated with the automated robotic system, appeared to be similar to the quality of the slides that were manually treated. Small differences in observations occurred in windows between duplicate samples themselves (i.e. within the same treatment method), as well as between the treatment methods (i.e. the same sample). Titres of PC and PS obtained with manual and robotic treatments are shown in Table 1. Differences in titres of one dilution of serial dilutions were statistically insignificant (Desmonts and Remington 1980; Strijack et al. 2004). As shown in Table 1 , most observations did not yield any significant difference in titre between manual and robotic treatments. The observations 3 , 8 and 11, however, showed significant titre differences in the positive sample slides. In observation 3 the lower titre in manual treatment can be explained by the accidental use of an older batch of positive sample slides. Observation 8 showed a lower reaction in manual treatment than in robotic treatment. This difference can be due to a large number of cells in the 
Table 1 Titre values for PC and PS slides following manual (m) and automated (a) procedure

\begin{tabular}{|c|c|c|c|c|c|c|c|}
\hline \multirow[t]{2}{*}{ Run } & \multicolumn{3}{|c|}{ Positive control slides (PC) } & \multicolumn{4}{|c|}{ Positive sample slides (PS) } \\
\hline & Titre $_{\mathrm{m}}$ & Titre $_{\mathrm{a} 1}$ & Titre $_{\mathrm{a} 2}$ & Titre $_{\mathrm{m} 1}$ & Titre $_{\mathrm{m} 2}$ & Titre $_{\mathrm{a} 1}$ & Titre $_{\mathrm{a} 2}$ \\
\hline 1 & 12,800 & 12,800 & 12,800 & 6,400 & 6,400 & 6,400 & 6,400 \\
\hline 2 & 6,400 & 6,400 & 12,800 & $1,600^{\mathrm{a}}$ & $800^{\mathrm{a}}$ & $800^{\mathrm{a}}$ & $800^{\mathrm{a}}$ \\
\hline 3 & 6,400 & 12,800 & 12,800 & $1,600^{\mathrm{b}}$ & $800^{\mathrm{b}}$ & 12,800 & 6,400 \\
\hline 4 & 12,800 & 12,800 & 12,800 & 3,200 & 3,200 & 3,200 & 3,200 \\
\hline 5 & 6,400 & 6,400 & 6,400 & $800^{\mathrm{a}}$ & $800^{\mathrm{a}}$ & $800^{\mathrm{a}}$ & $800^{\mathrm{a}}$ \\
\hline 6 & 12,800 & 12,800 & 12,800 & 3,200 & 6,400 & 3,200 & 6,400 \\
\hline 7 & 12,800 & 12,800 & 12,800 & 3,200 & 3,200 & 3,200 & 6,400 \\
\hline 8 & 6,400 & 6,400 & 6,400 & $800^{\mathrm{a}}$ & $800^{\mathrm{a}}$ & 3,200 & $1,600^{\mathrm{a}}$ \\
\hline 9 & 12,800 & 12,800 & 6,400 & $800^{\mathrm{a}}$ & $800^{\mathrm{a}}$ & $800^{\mathrm{a}}$ & $800^{\mathrm{a}}$ \\
\hline 10 & 6,400 & 6,400 & 6,400 & 3,200 & 3,200 & 6,400 & 6,400 \\
\hline 11 & 12,800 & 12,800 & 12,800 & 6,400 & 12,800 & 3,200 & 12,800 \\
\hline 12 & 12,800 & 12,800 & 6,400 & 3,200 & 3,200 & 3,200 & 3,200 \\
\hline
\end{tabular}

All PS slides contain two similar extracts; hence one slide yields two IF-judgements. The automated procedure involves two PC slides (one for each working area), whereas manual treatment involves only one PC.

${ }_{\mathrm{m}}$ Results referred to manual treatment.; ${ }_{\mathrm{a}}$ Results referred to automated treatment.

${ }^{a}$ Titres are lower as expected because of high amounts of cells in the samples (highly contaminated samples).

${ }^{\mathrm{b}}$ Titres are lower as expected because of accidentally used older batch of PS, yielding lower titres.

samples $\left(>10^{3}\right.$ per field of view). In observation 11 the significant difference between the two robotic treatment observations cannot be explained. These few diverging observations were therefore excluded in the comparison between robotic and manual treatments. Finally, NS processed by the automated system showed green specks more often, compared to manually treated NS in which specks were usually present at the border of the sample window. Microscopic observation of such slides, however, was not found to be impaired. The final test result for all NS slides was negative.

Average time needed for a maximum throughput run (144 determinations) was 2:55 $\pm 0: 05 \mathrm{~h}$ (based on six such runs). During 3 subsequent days, 288 slides per day were treated successfully with this procedure. During the test no problems with the robustness of the system were observed.

In our laboratory large-scale testing for bacterial brown rot and ring rot takes place, and therefore automation of the IF sample staining procedure could be desirable. In our study we focused on the automatic robotic staining of Rsol samples, assuming that for $\mathrm{Cms}$ conditions would not differ very much. Technically, with relatively minor software and hardware changes, the Tecan miniprep 75/2 system was indeed adapted by us to test two pathogens at a time (unpublished, present authors). For automated analysis of two pathogens the hardware of the Tecan miniprep $75 / 2$ had to be adapted so that the reservoir for the antibodies and that of the conjugate allowed each pipette tip to be placed in the correct liquid automatically. Furthermore a rack was constructed (replacing the standard serum container) with positions for eight tubes (one for each pipette tip), allowing separate but simultaneous application of the two antisera. in the same run. The positioning of the antiserum dilutions for two pathogens in separate tubes took more preparation time than when the standard container was used (and only one pathogen was tested in a run), and is therefore only suitable when high numbers of slides are processed. In a run with slides that were tested both for $\mathrm{Rsol}$ and $\mathrm{Cms}$ at the same time (20 slides, treated with both antisera and washed simultaneously) the system functioned flawlessly (unpublished results, present authors).

When processing $<72$ slides, manual staining may be more efficient, but as working with the automated system becomes routine, processing fewer number of samples by the automated robotic system may still be advantageous. Therefore the Tecan miniprep $75 / 2$ system was also adapted by making changes in the software programme process fewer slides in one run, but was restricted to processing a multiple of four slides at a time. 
Optimization of the automated system protocol (i.e. one extra washing step of $3 \mathrm{~min}$, followed by immediate blotting dry of slides) was necessary to practically eliminate the non-specific green specks, observed in samples under the fluorescence microscope. Spreading of drops of liquid over the entire slide windows presented occasional problems when using the robot, requiring the drops to be spread manually. This phenomenon, however, also occurs sporadically in manually stained samples. Based on high maximum throughput combined with less labour input and no differences in final IF results, we conclude that automated IF sample staining, using the Tecan miniprep $75 / 2$, is a reliable method for high-throughput of sample slides. The system is suitable for any large-scale screening that uses the immunofluoresence method. With the automated robotic system one person can stain 288 slides (288 samples with 2 different antisera or 576 samples for 1 antiserum) per day whereas with manual staining this would take 2 days of work.
Acknowledgements We would like to thank Mr. J. Voogd, C. E.A. Jochems, Ms. S. Somovilla Carrasco and Mrs. M. de Goffau for skilful technical assistance, H. de Bock, D. Bertens of Tecan Benelux B.V., Industrieweg 30, 4283 GZ Giessen, NL for adaptation of the Tecan Miniprep 75/2 robotic sample processor and its software and dr. ir. J. Schans for statistical advice.

\section{References}

Desmonts, G., \& Remington, J. S. (1980). Direct agglutination test for diagnosis of Toxoplasma infection: Method for increasing sensitivity and specificity. Journal of Clinical Microbiology, 11, 562-568.

Janse, J. D. (1996). Potato brown rot in western Europe History, present occurrence and some remarks on possible origin, epidemiology and control strategies. Bulletin OEPP, 26, 679-695.

Schans, J., \& Steeghs, M. H. C. G. (1998). Strategy and results of eradication of brown rot in The Netherlands. Bulletin OEPP, 28, 121-133.

Strijack, C., Harding, G. M. K., Ariano, R. E., \& Zelenitsky, S. A. (2004). Peritoneal Fluid Titer Test for Peritoneal Dialysis-Related Peritonitis. Antimicrobial Agents and Chemotherapy, 48, 1719-1726. 